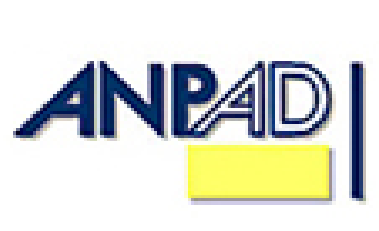

Disponível em http://www.anpad.org.br/rac

RAC, Curitiba, v. 15 , n. 4 , art. 3 , pp. 608-630, Jul./Ago. 2011

$(\mathrm{cc})$ EY-No

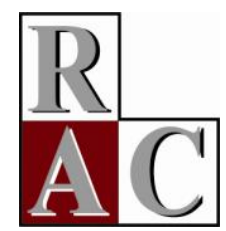

\title{
A Relação entre Confiança e Custos de Transação em Relacionamentos Interorganizacionais
}

The Relation between Trust and Transaction Costs in Interorganizational Relationships

Carlos Henrique Mascarenhas de Andrade*

E-mail: carlos.andrade@aero.bombardier.com Bombardier Aerospace Montreal, Canadá.

Sérgio Fernando Loureiro Rezende E-mail: srezende@pucminas.br Pontifícia Universidade Católica de Minas Gerais - PUC MINAS Belo Horizonte, MG, Brasil.

\author{
Márcio Antônio Salvato \\ E-mail: marcio.salvato@gmail.com \\ IBMEC-MG \\ Belo Horizonte, MG, Brasil.
}

Patrícia Bernardes E-mail: patib@pucminas.br Pontifícia Universidade Católica de Minas Gerais - PUC MINAS Belo Horizonte, MG, Brasil.

* Endereço: Carlos Henrique Mascarenhas de Andrade

5160, MacDonald Av. Ap 109, Cote Saint-Luc, Cidade: Montreal, Quebec/Canada, H3X2V8.

Copyright (C) 2011 RAC. Todos os direitos, até mesmo de tradução, são reservados. É permitido citar parte de artigos sem autorização prévia, desde que seja identificada a fonte. 


\title{
Resumo
}

Partindo do pressuposto de que a confiança é um mecanismo relacional de governança que pode interferir nos custos associados à formação, desenvolvimento e manutenção de relacionamentos interorganizacionais, buscase, neste trabalho, testar a relação entre confiança e custos de transação nesses relacionamentos. A partir da literatura de confiança e de custos de transação, foram formuladas quatro novas hipóteses, que sugerem uma relação negativa entre confiança e custos de informação, de negociação, de implementação e de monitoramento em relacionamentos interorganizacionais. Utilizando análise de regressão múltipla, tais hipóteses foram testadas em um estudo de caso de natureza quantitativa, em que o comprador é representado por uma montadora de automóveis e os fornecedores por uma amostra dos seus principais fornecedores. Os resultados sugerem que a confiança está relacionada negativamente com os custos de negociação e de monitoramento. Não foram encontradas, por sua vez, relações estatísticas significativas entre confiança e custos de informação e de implementação. Acredita-se que tais resultados representam uma compreensão mais refinada da relação entre confiança e custos de transação em relacionamentos interorganizacionais à medida que sugerem que os quatro tipos de custos de transação não são perfeitamente correlacionados.

Palavras-chave: confiança; custos de transação; relacionamentos interorganizacionais; indústria automotiva.

\begin{abstract}
Based on the assumption that trust is a mechanism of relational governance, which can influence the costs associated with the formation, development and maintenance of interorganizational relationships, in this article one aims to test the relation between trust and transaction costs in these relationships. Based on the literature on trust and on transaction costs, one puts forward four new hypotheses, which suggest a negative relation between trust and costs of information, of negotiation, of implementation and of monitoring in interorganizational relationships. These hypotheses were tested using multiple regression analysis in a quantitative case study where the buyer is illustrated by an automaker and its suppliers are represented by a sample of the major ones. The results suggest that there are significant, yet negative relations between trust and costs of negotiation and of monitoring. The relations between trust and costs of information and of implementation, in turn, are not statistically significant. We believe that these results represent a more-fined grained understanding of the relation between trust and transaction costs in interorganizational relationships to the degree that it is suggested that the four types of transaction costs are not perfectly correlated.
\end{abstract}

Key words: trust; transaction costs; interorganizational relationships; automotive industry. 


\section{Introdução}

A confiança em relacionamentos interorganizacionais, vista como importante mecanismo relacional de governança (Macaulay, 1963; MacNeil, 1978), tem chamado atenção de diversos pesquisadores estrangeiros (Gambetta, 1988; Kramer, 2006; McEvily, Perrone, \& Zaheer, 2003) e brasileiros (Cunha \& Melo, 2006; Lazzarini, Madalozzo, Artes, \& Siqueira, 2005), como alternativa aos contratos formais (Williamson, 1985; Zylbersztajn \& Sztajn, 2005). Entre os vários benefícios associados à confiança, destacam-se a possibilidade de mitigar incertezas sociais (Lazzarini, Miller, \& Zenger, 2008) e facilitar os processos de transferência de conhecimento entre os atores (Jensen \& Webster, 2009; Mesquita \& Lazzarini, 2008). Além disso, Dyer (1997), Dyer e Chuo (2000, 2003) e Mesquita e Brush (2008) propõem que a confiança tem o potencial de coibir comportamentos oportunistas e, com isso, minimizar custos de transação em relacionamentos interorganizacionais, especialmente naqueles que envolvem ativos altamente específicos (Argyres \& Mayer, 2007). Esse último ponto, a relação entre confiança e custos de transação em relacionamentos interorganizacionais é o tema deste trabalho.

A despeito de se reconhecer que relacionamentos interorganizacionais, pautados por confiança, têm efeitos econômicos importantes para as partes envolvidas (Pereira \& Luce, 2009; Smith \& Aldrich, 1991), é surpreendente a escassez de estudos empíricos que testam a relação entre confiança e custos de transação (Dyer \& Chuo, 2003, p. 57). Para esses últimos autores, tal escassez pode ser explicada pela dificuldade de operacionalizar esses dois construtos.

A fim de diminuir essa lacuna e tendo como ponto de partida os quatro artigos elaborados por Dyer e coautores (Dyer, 1997; Dyer \& Chuo, 2000, 2003; Dyer, Cho, \& Chu, 1998), busca-se testar a relação entre confiança e custos de transação nos relacionamentos interorganizacionais de uma montadora de automóveis com seus principais fornecedores, localizados no mercado brasileiro. Cabe salientar que, além do contexto institucional, este trabalho se distingue dos trabalhos que o inspiraram em três outros pontos. Primeiro, enquanto Dyer e coautores operacionalizam custos de transação a partir da diferenciação entre custos que são incorridos antes da transação (custos ex ante) e custos que acontecem após a transação (custos ex post), optou-se, a partir de Williamson (1985), por levar adiante tal distinção, desmembrando os custos ex ante em custos de informação e de negociação, enquanto os custos ex post são classificados em custos de implementação e de monitoramento. Segundo, a partir da revisão de literatura, ampliou-se o número de variáveis de controle de dois para sete, a saber: número de processos de compra, tempo de duração dos processos de compra, grau de complexidade de produtos e de processos, tempo de relacionamento, tamanho do fornecedor e volume econômico de transações. Terceiro, ao invés de uma survey junto a fornecedores, realizou-se um estudo de caso, de natureza quantitativa, de uma montadora e seus principais fornecedores. Assim, a relação entre confiança e custos de transação é testada neste trabalho sob o ponto de vista da montadora.

Para além dessa introdução, o presente artigo está organizado da seguinte forma. Na segunda seção, apresenta-se a revisão de literatura, a qual contempla uma discussão acerca dos construtos confiança e custos de transação para, em seguida, introduzir as hipóteses de pesquisa. Na terceira seção, detalha-se a metodologia. Os resultados de pesquisa são apresentados e discutidos na quarta seção, enquanto a seção final contém as conclusões, recomendações e limitações da pesquisa.

\section{Revisão de Literatura}

\section{Confiança}

Inicialmente, pode-se dizer que o tema confiança despertou interesse da comunidade acadêmica, a partir das décadas de 60 e 70 com os resultados de pesquisas oriundas, em sua maioria, da área da psicologia (Deutsch, 1958, 1960, 1962; Gabarro, 1978; Giffin, 1967; Rotter, 1967, 1971). Nas décadas 
seguintes, outros trabalhos de grande envergadura (Larzelere \& Huston, 1980; Lewis \& Weigert, 1985; Rotter, 1980) contribuíram para ampliar as bases teóricas desse construto, tanto na esfera interpessoal quanto no âmbito organizacional, o que delineou de forma mais detalhada diversas acepções quanto à natureza da confiança, tais como: expectativa (Rotter, 1967; Zucker, 1986); crença (Sitkin \& Roth, 1993); estado psicológico (Rousseau, Stikin, Burt, \& Camerer, 1998); percepção (Doney \& Cannon, 1997); assunção (Robinson, 1996); disposição (para assumir riscos) - (Mayer, Davis, \& Schoorman, 1995); e probabilidade subjetiva (Gambetta, 1988; Nooteboom, 1996). Em que pese essa multiplicidade de acepções, neste trabalho confiança é geralmente entendida como a existência de uma expectativa ou crença positiva, mantida por um ator quanto à integridade (Barber, 1983; Rotter, 1980); benevolência (Deutsch, 1962); e/ou competência (Nooteboom, 1996) de outrem.

A partir deste conceito de confiança e tendo em vista a profusão de trabalhos realizados no âmbito da confiança em relações interpessoais (Larzelere \& Huston, 1980); intraorganizacionais (Zanini, Lusk, \& Woff, 2009); e interorganizacionais (Cunha \& Melo, 2006), esta seção está dedicada a detalhar os três elementos que, a partir da sugestão de Mayer, Davis e Schoorman (1995), constituíram o construto de confiança, testado neste trabalho: integridade, benevolência e competência.

Para Gabarro (1978), a integridade é um dos principais elementos da confiança, sendo baseado na aderência do comportamento de um ator a um determinado conjunto de normas comumente aceitas como justas e corretas pelo grupo de atores a que pertence. $\mathrm{O}$ ator íntegro apresenta atos coerentes com suas palavras e pauta-se pela retidão de caráter e obediência a princípios morais. Mas o ator íntegro não é necessariamente confiável, porquanto, ainda que respeite regras morais e cumpra promessas, pode não ser detentor de benevolência (Larzelere \& Huston, 1980).

Esse segundo elemento, benevolência, é definido como característica própria daquele que se interessa genuinamente pelo bem-estar do outro, ainda que isto não lhe gere compensação ou vantagem direta (Deutsch, 1962). Outros termos, como altruísmo (Sober \& Wilson, 1998) e lealdade (Butler \& Cantrell, 1984) são utilizados em acepções semelhantes.

O terceiro elemento, competência, ganha especial proeminência na esfera organizacional (Nooteboom, 1996), sendo definido como capacidade técnica ou operacional, conhecimento sobre determinado assunto, habilidade interpessoal ou de negociação, bem como discernimento para oferecer soluções a situações inesperadas (Gabarro, 1978; Moorman, Deshpande, \& Zaltman, 1993). Ou seja, trata-se do conjunto de capacidades ou habilidades sobre determinado assunto que qualifica um ator para a execução de uma ação ou tarefa determinada (Butler, 1991). Assim, a competência parece ser de extrema relevância para a geração de expectativa de que um ator irá cumprir aquilo que se propôs a realizar, não apenas porque seja íntegro ou benevolente, mas porque tem efetivamente a capacidade técnica ou conhecimento para fazê-lo.

\section{Custos de transação}

Inicialmente, pode-se dizer que os custos de transação têm relevante impacto na eficiência econômica das nações (North, 1990) e/ou das firmas (Williamson, 1991). Estimativas sugerem que tais custos possam representar tanto quanto $35 \%$ a $40 \%$ dos custos associados à atividade econômica mundial (North, 1990). Introduzido por Coase (1937) e posteriormente sedimentado por Williamson (1985, 1991, 1996) sob a denominação Economia de Custos de Transação (ECT), a concepção de custos de transação se tornou, ao longo das últimas décadas, um dos principais arcabouços teóricos na área organizacional (Geyskens, Sttenkamp, \& Kumar, 2006). Interessa-nos aqui a distinção entre custos de transação ex ante e custos de transação ex post, tendo em vista que a operacionalização de custos de transação, neste trabalho, está calcada nesta diferenciação.

Posto isto, entendem-se como custos de transação ex ante aqueles anteriores e preparatórios da transação. Ou seja, são os custos envolvidos em levantar informações acerca da transação, bem como em criar salvaguardas aos contratos firmados (Dyer, 1997). Já os custos de transação ex post se referem aos custos de possíveis adaptações do contrato original e de vigilância do cumprimento das 
cláusulas do acordo firmado entre os atores (Williamson, 1985). Envolvem também os custos de reparação de danos decorrentes de ações indevidas e das perdas associadas a tais ações.

No primeiro tipo de custos, ex ante, têm-se os custos de informação, que podem ser identificados como os primeiros custos incorridos em uma transação (Demsetz, 1991). Referem-se aos custos relacionados à busca de possíveis parceiros para efetuar transações e/ou custos na busca de informações adicionais, em casos de repactuação contratual (Cai, 2004).

Assim que os atores tenham chegado a um estado de menor incerteza entre si, há uma tendência de evolução da transação para a negociação (Dyer, 1997), envolvendo, portanto, a definição de obrigações, salvaguardas, responsabilidades e ganhos. Não raro, esses custos são provenientes de assessoria jurídica na formalização do contrato (Foss \& Foss, 2005). Por essas razões, normalmente há a geração de custos de transação que são identificados como custos de negociação (Williamson, 1985).

A negociação termina com a efetiva decisão quanto ao estabelecimento do acordo entre os atores, normalmente feita com base nas informações levantadas e trocadas entre si e nas condições até então negociadas (Demsetz, 1991). Esse é o momento em que, teoricamente, se diz que a transação ocorre (Williamson, 1991) e marca, portanto, a divisão entre os custos de transação ex ante e os custos de transação ex post (Williamson, 1985).

O primeiro tipo de custos de transação ex post denominam-se custos de implementação e se refere aos custos envolvidos na execução inicial do contrato firmado entre os atores (Dyer, 1997). Nesse momento, há, normalmente, nova e extensa abertura de informações (comumente as mais valiosas), que os atores utilizam para detalhar e colocar em prática o que até então eram cláusulas contratuais (Madhok, 2002), implicando, normalmente, aprendizagem mútua (Vanneste \& Puranam, 2010). Envolvem também custos de barganha entre os atores, à medida que algumas cláusulas contratuais necessitam de adaptação (Dyer \& Chuo, 2003).

No entanto, os esforços dos atores para a redução progressiva da assimetria de informação, para a definição de critérios e condições contratuais e para o desdobramento e detalhamento dessas condições não conseguem eliminar, por completo, as incertezas sociais e/ou do valor de troca das transações (Lazzarini, Miller, \& Zenger, 2008). Isso implica, segundo Williamson (1993), a inevitável necessidade de vigilância para que os atores possam prevenir-se de comportamentos oportunistas e/ou inadequados e, eventualmente, o uso de sanções e de iniciativas de recuperação ou de correção de perdas decorrentes de tais comportamentos. Williamson (1985) denomina os custos associados ao controle da transação de custos de monitoramento.

\section{Hipóteses de pesquisa}

De maneira geral, sugere-se uma relação negativa entre confiança e custos de transação ex ante (custos de informação e de negociação) e ex-post (custos de implementação e de monitoramento) em relacionamentos interorganizacionais (Dyer, 1997; Dyer \& Chuo, 2000, 2003; Dyer, Cho, \& Chu, 1998). Tal relação pode ser mais bem entendida por meio da formulação de quatro novas hipóteses.

A primeira hipótese aponta uma relação inversa entre confiança e custos de informação. Acredita-se que em relacionamentos interorganizacionais, governados por níveis mais elevados de confiança, os atores tendem a economizar tempo na busca de informações (Dyer, 1997), já que essas são normalmente fornecidas em primeira mão pelos atores diretamente envolvidos, sem a necessidade de intermediários e/ou de maiores esforços para sua obtenção (Cai, 2004). Além disso, sugere-se que nesses relacionamentos há menor necessidade de checagens adicionais para garantir a veracidade ou precisão das informações, uma vez que os atores tendem a acreditar que as informações fornecidas são corretas, precisas e valiosas (Macaulay, 1963). Com base neste raciocínio, formula-se a primeira hipótese da seguinte forma:

H1: Ceteris paribus, existe uma relação negativa entre confiança e custos de informação em relacionamentos interorganizacionais. 
A segunda hipótese do trabalho propõe uma relação negativa entre confiança e custos de negociação (Zaheer, McEvily, \& Perrone, 1998). Em relacionamentos interorganizacionais, caracterizados por níveis mais elevados de confiança, sugere-se que os atores possuem maior disposição para contratar mesmo, na ausência de salvaguardas adequadas ou contratos formais (Macaulay, 1963), o que, em última instância, tem impactos positivos na redução dos custos de negociação, principalmente no que diz respeito aos ônus de assessoria jurídica (Dyer \& Chuo, 2003). Ademais, verifica-se que, nesses relacionamentos, os atores tendem a ser mais flexíveis no decorrer do processo de barganha (Dyer, 1997). Isso porque tal processo é usualmente pautado pela crença de que as partes contribuirão, de maneira equânime, para o desenvolvimento do relacionamento (Dyer \& Chuo, 2003). Assim, ao se concentrar em gerar valor a partir do relacionamento (Pereira \& Luce, 2009) - ao invés de somente se preocupar com a possibilidade de apropriação indébita na distribuição de ganhos - (Puranam \& Vanneste, 2009), os atores podem beneficiar-se de redução nos custos de negociação. Tendo em vista essa discussão, enuncia-se formalmente a segunda hipótese.

H2: Ceteris paribus, existe uma relação negativa entre confiança e custos de negociação em relacionamentos interorganizacionais.

Tal como as duas primeiras hipóteses do trabalho, a terceira sugere uma relação negativa entre confiança e custos de implementação. Tendo em vista que os contratos são, em função da racionalidade limitada dos atores, incompletos (Williamson, 1985; Zylbersztajn \& Sztajn, 2005), existem, teoricamente, oportunidades para o exercício de comportamentos oportunistas no decorrer da sua implementação (Williamson, 1993). Isso implica maiores custos de transação, que podem ser traduzidos em termos de redesenho de contratos, custos adicionais de barganha e acordo entre os atores (Puranam \& Vanneste, 2009; Vanneste \& Puranam, 2010). Nesse sentido, em relacionamentos em que maiores níveis de confiança se fazem presentes, tais custos podem ser minorados à medida que os atores tendem a não recorrer a medidas adicionais de proteção de seus interesses para suprimirem eventuais lacunas contratuais (Dyer, 1997). Além disso, na ocorrência de situações não originalmente previstas, é possível que os atores se vejam compelidos a novas rodadas de negociação (Dyer \& Chuo, 2003). A forma de lidar com essas situações e a convergência para soluções satisfatórias podem ser beneficiadas em relacionamentos pautados por níveis mais elevados de confiança, à medida que os atores tendem a assumir que as ações ou proposições feitas são pautadas por princípios de integridade e benevolência, facilitando, assim, eventuais ajustes (Uzzi, 1997). Como resultado, tem-se que os custos de implementação tendem a ser menores. Essa discussão nos leva à formulação da terceira hipótese.

H3: Ceteris paribus, existe uma relação negativa entre confiança e custos de implementação em relacionamentos interorganizacionais.

Finalmente, a quarta hipótese postula uma relação negativa entre confiança e custos de monitoramento em relacionamentos interorganizacionais. Níveis mais elevados de confiança nesses relacionamentos tendem a desestimular os atores a se engajarem em ações que têm como objetivo checar se algum deles eventualmente oculta ou distorce informações, ou mesmo faz propositadamente uso de ações que buscam maximizar ganhos individuais em detrimento do relacionamento (Bromiley \& Cummings, 1995). Ao colocar em segundo plano ações que visam verificar e coibir o oportunismo alheio, os atores podem beneficiar-se de redução nos custos de monitoramento (Dyer \& Chuo, 2003). Além disso, em relacionamentos pautados por níveis mais elevados de confiança, existe maior predisposição dos atores em acreditar que ambos são competentes para desempenhar aquilo que foi previamente acordado (Butler \& Cantrell, 1984). Essa crença ou conhecimento acerca da competência dos atores pode levar à redução de atividades de monitoramento e, consequentemente, dos custos a elas associados, pelo fato de que essas são desnecessárias. Essa discussão fornece a base para a formulação da última hipótese.

H4: Ceteris paribus, existe uma relação negativa entre confiança e custos de monitoramento em relacionamentos interorganizacionais. 


\section{Metodologia}

Escolheu-se como método de pesquisa o estudo de caso de natureza quantitativa (Dubois \& Gibbert, 2010; Yin, 1994). Como o objetivo do trabalho implica testar a relação entre os construtos confiança e custos de transação, sob o ponto de vista do comprador, justifica-se a escolha de tal método pela necessidade de selecionar uma firma compradora que se relacionasse com um número razoável de fornecedores de primeira linha para que, dessa forma, fosse possível coletar dados que permitissem conduzir testes estatísticos de natureza inferencial (Babbie, 2005). Ademais, a necessidade de se minimizar a interferência de variáveis associadas às características particulares de cada firma reforçou a nossa opção pelo estudo de caso único de natureza quantitativa; entretanto tal opção, tal como reconhecido nas limitações do trabalho, interfere substancialmente na possibilidade de generalização dos resultados, já que esses podem, eventualmente, ser idiossincráticos da firma pesquisada.

Após a definição do método de pesquisa, partiu-se para a seleção do caso que, neste trabalho, é exemplificado por uma montadora de automóveis e seus principais fornecedores. Tal como Dyer e colegas (Dyer, 1997; Dyer \& Chuo, 2003; Dyer et al., 1998) e Sako e Helper (1998), acredita-se que a indústria automotiva representa contexto adequado para o teste das hipóteses de pesquisa, à medida que suas características estruturais propiciam o desenvolvimento de relacionamentos interorganizacionais, em que há elevada possibilidade de ocorrer variação de níveis de confiança e montante de custos de transação (Wasti \& Wasti, 2008). No que tange à montadora selecionada neste trabalho, doravamente denominada AUTOMAKER, questões relacionadas à facilidade de acesso e conhecimento prévio dos pesquisadores sobre a firma determinaram a escolha do caso propriamente dito.

O instrumento de coleta de dados utilizado foi o questionário semiestruturado (Babbie, 2005) composto por três seções. A primeira mede a existência e a gradação do construto confiança, a partir de doze assertivas, algumas positivas outras negativas, quanto à percepção do comprador sobre o comportamento dos fornecedores (ver Tabela 1). Para tanto foi utilizada a escala LIKERT de cinco pontos (Babbie, 2005), com a seguinte gradação: 1-discordo totalmente; 2-discordo parcialmente; 3 nem discordo, nem concordo; 4- concordo parcialmente; 5-concordo totalmente. Cabe destacar que, tal como Zaheer, McEvily e Perrone (1998), Dyer e Chuo (2003) e Gulati e Nickerson (2008), essa alternativa de mensurar confiança significa atentar para as consequências e não para os antecedentes da confiança. Nesse sentido, as assertivas dos elementos de confiança dizem respeito à magnitude ou níveis de confiança entre comprador e fornecedor. Gulati e Sytch (2008, p. 166) pontuam que tal procedimento implica considerar confiança como exógena ao relacionamento interorganizacional. 
Tabela 1

\section{Assertivas dos Elementos de Confiança: Primeiro Questionário}

\begin{tabular}{l}
\hline \multicolumn{1}{c}{ Elemento: Integridade } \\
\hline - Meu FORNECEDOR DE MAIOR APV se comporta de forma verdadeira em nossas relações (Integridade 1) \\
- Se meu FORNECEDOR DE MAIOR APV tivesse a chance, certamente tiraria vantagens de \\
vulnerabilidades de minha empresa (Integridade 2) \\
- Meu FORNECEDOR DE MAIOR APV tem um forte senso de justiça na condução de nosso relacionamento \\
(Integridade 3) \\
- Meu FORNECEDOR DE MAIOR APV cumpre com suas promessas (Integridade 4) \\
\hline
\end{tabular}

\section{Elemento: Benevolência}

- Meu FORNECEDOR DE MAIOR APV deseja o melhor para minha empresa, mesmo que isto não o beneficie diretamente. (Benevolência 1)

- Eu sei que posso contar com meu FORNECEDOR DE MAIOR APV para me ajudar se minha empresa tiver dificuldades. (Benevolência 2)

- Meu FORNECEDOR DE MAIOR APV tomaria ações nocivas contra minha empresa, mesmo que estas pudessem ser evitadas. (Benevolência 3)

- No momento de tomada de decisões, meu FORNECEDOR DE MAIOR APV se preocupa com o que é bom para minha empresa. (Benevolência 4)

\section{Elemento: Competência}

- O meu FORNECEDOR DE MAIOR APV desempenha suas atividades de forma correta. (Competência 1)

- Não tenho quaisquer dúvidas de que meu FORNECEDOR DE MAIOR APV tem competência para garantir o sucesso de projetos importantes nos quais minha empresa esteja envolvida. (Competência 2)

- Meu FORNECEDOR DE MAIOR APV tem total capacidade (técnica, interpessoal) para cumprir aquilo que dele se espera em nossas relações. (Competência 3)

- Eu não preciso conferir o trabalho efetuado por meu FORNECEDOR DE MAIOR APV para ter certeza de que ele o desempenhou corretamente. (Competência 4)

A segunda parte do questionário se refere à mensuração dos custos de transação. A partir de Dyer e Chuo (2003), mediram-se tais custos com base no tempo de dedicação dos compradores às ações diretamente envolvidas nas transações com os principais fornecedores. Precedida de um exemplo, que tinha como propósito fornecer orientações sobre como responder à questão, esta foi formulada da seguinte maneira: Indique nos espaços apropriados a seguir, qual o percentual de tempo em média que é gasto para cada uma das atividades detalhadas pelos números 1, 2, 3 e 4 na comparação com o tempo total de sua semana de trabalho.

1. Ações de Informação: Consultas e troca de informações iniciais com o Fornecedor sobre ()\% projetos, desenhos, datas chave (RFQ, Technical Reviews, recebimento de propostas).

2. Ações de Negociação: Negociação comercial, discussão e análise de custos, discussão de ( )\% condições comerciais, cláusulas contratuais, planos de desempenho, alinhamento geral para recomendação.

3. Ações de Implementação: Detalhamento de cronogramas e planos de trabalho, realização de ( )\% cadastros, emissão e envio de pedidos, reuniões de Kickoffs, detalhamento de ferramentais para liberação de verbas e pedidos.

4. Ações de Monitoramento e Reforço de Contratos: Monitoramento e gestão do contrato, ( )\% acompanhamento e revisão de cronogramas, ajustes e renegociação de condições e situações não previstas, modificações do projeto, reforço para cumprimento de condições previamente pactuadas. 
Finalmente, a terceira parte do questionário compreende um conjunto de perguntas que visa aferir as sete variáveis de controle do presente estudo. As duas primeiras têm a ver com o número de compras conduzido pelos compradores no espaço de doze meses e seu tempo médio de processamento (Wasti \& Wasti, 2008), isso porque determinado fornecedor pode ter um número mais elevado de processos de compra (ou processos de compra mais longos), o que, teoricamente, tem o potencial de elevar custos de transação ex ante e/ou ex post, sem que isso tenha ligação com o nível de confiança. Outras duas variáveis de controle se referem ao grau de complexidade dos produtos e o grau de complexidade dos processos relacionados aos fornecedores, ambas medidas por escala LIKERT de cinco pontos (Babbie, 2005), com a seguinte gradação: 1-muito simples; 2-simples; 3-média complexidade; 4-complexo; 5-muito complexo. Acredita-se que o grau de complexidade dos produtos possa interferir no tempo gasto quanto à troca de informações e implementação dos contratos, enquanto a complexidade dos processos pode afetar o tempo gasto na negociação e monitoramento dos contratos (Gulati \& Nickerson, 2008).

Controlou-se também o tempo de relacionamento, o qual visa eliminar a influência da curva de aprendizado inerente aos relacionamentos interorganizacionais (Lewicki, Macallister, \& Bies, 1998), bem como dos benefícios comumente associados a relacionamentos de mais longo prazo, tais como negociações mais fluidas e sistemas de produção mais eficientes (Mesquita \& Brush, 2008). Para a mensuração dessa variável indagou-se o tempo aproximado de relacionamento da montadora com o fornecedor em foco.

Em seguida, buscou-se controlar os efeitos do tamanho do fornecedor (Dyer \& Chuo, 2003), medido pelo número médio de empregados, à medida que fornecedores de maior porte podem ter procedimentos de contratação mais longos, maior número de pessoas envolvidas e departamentalização mais avançada, o que, por sua vez, podem afetar os custos de transação. Finalmente, a última variável de controle deste estudo com potencial para interferir nos custos de transação é o volume econômico de compras transacionado anualmente (Dyer \& Chuo, 2003). No nosso estudo essa variável foi medida pelo APV (Annual purchased value - valor comprado anualmente), que é a métrica utilizada pela AUTOMAKER para indicar o volume de compras negociado entre a montadora e determinado fornecedor.

Quando a primeira versão do questionário ficou pronta, em outubro de 2007, localizou-se, dentro da AUTOMAKER, um grupo piloto de 10 indivíduos que já haviam atuado na posição de comprador para o teste do questionário. Findo o pré-teste e incorporadas as sugestões recebidas, partiu-se para a definição dos respondentes do questionário que, no nosso trabalho, ficaram representados pelos 26 compradores da AUTOMAKER, isso porque, na firma pesquisada, são esses indivíduos que possuem estreita ligação com os fornecedores, sendo responsáveis por atividades relacionadas à formulação, implementação e monitoramento de contratos (Argyres \& Mayer, 2007).

Assim, solicitamos a cada comprador que respondesse aos questionários acerca de seus dois principais fornecedores, mensurados por ordem decrescente de APV. Os dois questionários foram aplicados em semanas separadas, em segundas-feiras, sendo o primeiro no dia 10 de dezembro de 2007 e o segundo no dia 17 seguinte. Os compradores receberam as instruções pessoalmente, foi-lhes garantido o anonimato quanto às respostas e foi-lhes dada uma semana de prazo para a resposta. Ademais, tomou-se o cuidado de esperar o retorno do primeiro para efetuarmos a aplicação do segundo a fim de evitar respostas seriadas e descompromissadas. De um total de 26 questionários distribuídos na primeira rodada, obteve-se um retorno de $100 \%$, enquanto na segunda rodada, esse percentual caiu para 92\%, o que totalizou, ao final da coleta de dados, uma amostra de 50 questionários válidos, correspondendo a compradores distintos. Cabe salientar que, apesar de a amostra final ser considerada pequena, conseguiu-se atingir o número de 50 que, segundo Hair, Anderson e Tatham (1988), é o número mínimo para executar uma análise fatorial. Com os questionários coletados e validados, procedeu-se, finalmente, à análise de dados, a qual foi balizada pelos seguintes critérios. Primeiramente, fez-se uso de estatística descritiva para todas as variáveis mediante a utilização de média, mediana e outras medidas de dispersão (Malhotra, 2001; Rea \& Parker, 2000). Em seguida tentou-se reduzir o número das variáveis constituintes do construto confiança via análise fatorial ou componentes principais (Hair, Anderson, \& Tatham, 1988). No caso 
deste estudo, foi utilizada a análise fatorial exploratória, balizada pelos seguintes critérios. O tamanho da amostra deve ser de, pelo menos, 50 casos (Hair et al., 1988). Além disso, as cargas fatoriais devem ultrapassar 0.50 para serem consideradas significativas. Mingoti (2005) ainda ressalta que as comunalidades devem evidenciar percentuais superiores a 0.50 e que a medida de adequação da amostra de Kaiser-Meyer-Olkim (KMO) deve apresentar índices superiores a 0.50. Finalmente, a análise fatorial requer que o Alfa de Cronbach ultrapasse o valor de 0.70 (Malhotra, 2001).

Em seguida, procedeu-se à análise de regressão multivariada (Elian, 1998; Hair et al., 1988). Para tanto a técnica utilizada foi a regressão linear múltipla (Elian, 1998), pois a variável dependente era contínua e as variáveis independentes eram discretas ou contínuas. $\mathrm{O}$ modelo escolhido foi o stepwise (Elian, 1998).

Neste modelo, a primeira etapa compreendeu a verificação do coeficiente de determinação de regressão $\left(\mathrm{R}^{2}\right)$ em regressão linear simples. Com amostra de 50 casos e nível de significância mínimo de $95 \%$, foram consideradas somente as variáveis com valores $\mathrm{R}^{2}$ superiores a $20 \%$ de explicação da regressão. $\mathrm{O}$ segundo passo do modelo compreendeu o teste de multicolinearidade, por meio da medida VIF (Variance Inflation Fator), para as variáveis que alcançaram o patamar definido na etapa anterior. Hair et al. (1988) sugerem um valor de 10.0 para estabelecer o limiar da multicolinearidade. Como terceiro e último passo do modelo stepwise, foram calculados, para as variáveis que não apresentaram multicolinearidade, os coeficientes de regressão padronizados, também chamado Beta, em que se comparou diretamente o efeito parcial de cada variável independente na variável dependente. Neste estudo, utilizou-se o intervalo de confiança de $95 \%$ e margem de erro de $5 \%$ para testar a significância dos valores dos coeficientes (Betas).

\section{Resultados}

\section{Resultados de estatística descritiva}

O índice de confiança geral, calculado por meio da média obtida nas 12 assertivas que tratam dos elementos de confiança (Doney \& Cannon, 1997), foi de 3,26 com desvio padrão de 1,13, o que significa nível razoável de confiança da AUTOMAKER em relação aos seus principais fornecedores. Quanto esse resultado é detalhado em termos dos elementos de confiança, observa-se que a competência foi o que obteve a maior média $(X=3,58)$, indicando, portanto, que os fornecedores da AUTOMAKER são vistos como sendo mais competentes quando comparados com os elementos benevolência e integridade.

Tabela 2

Média da Confiança Geral e seus Elementos

\begin{tabular}{lccc}
\hline \multicolumn{1}{c}{ Elementos de Confiança } & Média & Mediana & Desvio-padrão \\
\hline Confiança Geral & 3,26 & 4 & 1,13 \\
Benevolência & 3,26 & 4 & 1,08 \\
Competência & 3,58 & 4 & 1,13 \\
Integridade & 2,93 & 3 & 1,11 \\
\hline
\end{tabular}

Nota. Fonte: Dados da pesquisa.

Os resultados da variável custos de transação mostram que, de maneira geral, do total de horas trabalhadas numa semana típica, mais de $45 \%$ do tempo dos compradores é dedicado às atividades de relacionamento com os principais fornecedores da AUTOMAKER. Deste tempo total, as ações que geram custos de negociação correspondem a uma média de $15,89 \%$, enquanto as de monitoramento 
possuem uma média de $12,73 \%$. Tal resultado sinaliza que os principais custos de transação incorridos pela AUTOMAKER dizem respeito a negociar e monitorar os seus principais fornecedores (ver Tabela 3).

Tabela 3

Resultados de Média, Mediana e Desvio-padrão dos Tipos de Custos de Transação

\begin{tabular}{lccc}
\hline \multicolumn{1}{c}{ Tipo de Custo } & Média & Mediana & Desvio-padrão \\
\hline Informação & $8,80 \%$ & $8 \%$ & $5,85 \%$ \\
Negociação & $15,89 \%$ & $15 \%$ & $7,60 \%$ \\
Implementação & $7,94 \%$ & $6 \%$ & $4,52 \%$ \\
Monitoramento & $12,73 \%$ & $11 \%$ & $8,24 \%$ \\
\hline
\end{tabular}

Nota. Fonte: Dados da pesquisa.

Os resultados das variáveis de controle indicam o perfil dos compradores investigados, o qual pode ser descrito da seguinte maneira. Respondem por $56 \%$ do volume total de compras da montadora. Em 2007, o volume de vendas para a AUTOMAKER de 92\% desses compradores superou 20 milhões de reais. São compradores de grande porte, já que a média geral do número de funcionários é de 1.121. Para 84\% dos compradores, o faturamento médio anual em 2007 situou-se entre 20 e 160 milhões. No que se refere ao tempo de relacionamento, a média é de 17 anos; $46 \%$ dos compradores transacionam com a AUTOMAKER há mais de 20 anos. Finalmente, a frequência média de processos de compra desses fornecedores com a montadora é de 11 processos por ano, com duração média de 3,15 meses por processo.

\section{Resultados de estatística inferencial}

Inicialmente efetuou-se uma análise fatorial exploratória da variável confiança, a fim de verificar a possibilidade de reduzir o número de itens que compunham essa variável. Ao calcular a correlação par-a-par de todos esses itens, observamos que, das 66 correlações obtidas, 38 (58\%) apresentam nível de confiança superior a $95 \%$ ( $\mathrm{p}$-valor $<0,05$ ), o que sugere nível significativo de identidade entre os itens e, portanto, a indicação da viabilidade da análise fatorial exploratória (ver Tabela 4). Cabe salientar que o Alfa de Cronbach, medido em relação às escalas somadas, atingiu 0,83 , revelando elevada confiabilidade das escalas utilizadas (Hair et al., 1988). 
Tabela 4

Resultados da Análise de Correlação dos Itens de Confiança

\begin{tabular}{|c|c|c|c|c|c|c|c|c|c|c|c|c|c|}
\hline & & B1 & $\mathrm{C} 1$ & I1 & B2 & I2 & $\mathrm{C} 2$ & B3 & I3 & C3 & B4 & I4 & $\mathrm{C} 4$ \\
\hline \multirow{2}{*}{ B1 } & Corr & - & & & & & & & & & & & \\
\hline & $\mathrm{P}$ & - & & & & & & & & & & & \\
\hline \multirow{2}{*}{$\mathrm{C} 1$} & Corr & 0,4754974 & - & & & & & & & & & & \\
\hline & $\mathrm{P}$ & $0,000277^{*}$ & - & & & & & & & & & & \\
\hline \multirow{2}{*}{ I1 } & Corr & 0,6367750 & 0,5148837 & - & & & & & & & & & \\
\hline & $\mathrm{P}$ & $0,000004^{*}$ & $0,000076^{*}$ & - & & & & & & & & & \\
\hline \multirow{2}{*}{ B2 } & Corr & 0,2557391 & 0,4416268 & 0,3985267 & - & & & & & & & & \\
\hline & $\mathrm{P}$ & $0,038066^{*}$ & $0,000745^{*}$ & $0,002282 *$ & - & & & & & & & & \\
\hline \multirow{2}{*}{$\mathrm{I} 2$} & Corr & 0,1786606 & 0,4539873 & 0,4333043 & 0,4025516 & - & & & & & & & \\
\hline & $\mathrm{P}$ & 0,1096766 & $0,000525^{*}$ & $0,000935^{*}$ & $0,002068^{*}$ & - & & & & & & & \\
\hline \multirow{2}{*}{$\mathrm{C} 2$} & Corr & 0,1229710 & 0,2798013 & 0,1414749 & 0,2533082 & 0,1264706 & - & & & & & & \\
\hline & $\mathrm{P}$ & 0,1999564 & $0,025759 *$ & 0,1661112 & $0,039531 *$ & 0,1932657 & - & & & & & & \\
\hline \multirow{2}{*}{ B3 } & Corr & 0,1015717 & 0,4013286 & 0,2749744 & 0,4186379 & 0,3554216 & 0,1083250 & - & & & & & \\
\hline & $\mathrm{P}$ & 0,2437011 & $0,002131 *$ & $0,027927 *$ & $0,001378^{*}$ & $0,006102 *$ & 0,2293819 & - & & & & & \\
\hline \multirow{2}{*}{ I3 } & Corr & 0,4261050 & 0,5294855 & 0,7326693 & 0,5061130 & 0,4350624 & 0,1784656 & 0,3684408 & - & & & & \\
\hline & $\mathrm{P}$ & $0,001133^{*}$ & $0,000045^{*}$ & $0,000000 *$ & $0,000103^{*}$ & $0,000892 *$ & 0,1099324 & $0,004595 *$ & - & & & & \\
\hline \multirow{2}{*}{$\mathrm{C} 3$} & Corr & 0,0182642 & 0,2791783 & 0,0034259 & 0,2776171 & 0,0657827 & 0,5948980 & 0,0190526 & 0,1062450 & - & & & \\
\hline & $\mathrm{P}$ & 0,4504363 & $0,026031 *$ & 0,4906805 & $0,026722 *$ & 0,3266876 & $0,000003^{*}$ & 0,4483081 & 0,2337428 & - & & & \\
\hline \multirow{2}{*}{ B4 } & Corr & 0,2425991 & 0,2462429 & 0,4301610 & 0,6056532 & 0,2753075 & 0,2381359 & 0,3901127 & 0,6608702 & 0,1006004 & - & & \\
\hline & $\mathrm{P}$ & $0,046522 *$ & $0,044043 *$ & $0,001018 *$ & 0,0000020 & 0,0277729 & 0,0497052 & $0,002794 *$ & $0,000001 *$ & 0,2457984 & - & & \\
\hline \multirow{2}{*}{ I4 } & Corr & 0,2792118 & 0,4331609 & 0,3864019 & 0,2721135 & 0,5446506 & 0,3311755 & 0,3214704 & 0,4130292 & 0,1952193 & 0,3342048 & - & \\
\hline & $\mathrm{P}$ & $0,026016^{*}$ & $0,000939 *$ & $0,003049 *$ & 0,0292798 & 0,0000261 & 0,0100520 & $0,012149 *$ & $0,001591 *$ & 0,0894366 & $0,009463^{*}$ & - & \\
\hline \multirow{2}{*}{$\mathrm{C} 4$} & Corr & 0,0974215 & 0,2855030 & $-0,0049838$ & 0,2217911 & 0,0643620 & 0,1292148 & 0,1404301 & 0,2664930 & 0,2340889 & 0,2445528 & 0,4736676 & - \\
\hline & $\mathrm{P}$ & 0,2527272 & $0,023376^{*}$ & 0,4864440 & 0,0628100 & 0,3302006 & 0,1881131 & 0,1679208 & $0,032090 *$ & 0,0527341 & 0,0451803 & $0,000293^{*}$ & - \\
\hline
\end{tabular}

Nota. * Resultados significativos a $95 \%$ de confiança

Fonte: Dados da pesquisa 
Além disso, a medida KMO evidenciou um valor de 0,71, enquanto o teste de esfericidade de Bartlett rejeitou a existência de correlações iguais a zero entre os itens com valores significativos para p-valor $<0,05$, apontando, portanto, que a matriz de correlações continha associações importantes entre os itens.

Para avaliar os fatores a serem extraídos para a análise fatorial, foram utilizados autovalores superiores a um (Hair et al., 1988). Na Tabela 5, pode-se verificar que apenas quatro fatores apresentaram autovalores superiores a essa medida, com percentual acumulado de variância de $70,11 \%$. Este resultado aponta a possibilidade de redução dos itens estudados em quatro fatores ortogonais, para expressar as informações relevantes do construto confiança.

Tabela 5

\section{Resultados da Análise Fatorial}

\begin{tabular}{cccc}
\hline Fator & Autovalores & Variância (percentual) & Percentual cumulativo \\
\hline $\mathbf{1}$ & 4,579702596 & 38,1641883 & 38,1641883 \\
$\mathbf{2}$ & 1,639256022 & 13,66046685 & 51,82465515 \\
$\mathbf{3}$ & 1,152910945 & 9,607591211 & 61,43224636 \\
$\mathbf{4}$ & 1,042289521 & 8,685746009 & 70,11799237 \\
$\mathbf{5}$ & 0,940451793 & 7,837098273 & 77,95509064 \\
$\mathbf{6}$ & 0,702564683 & 5,854705691 & 83,80979633 \\
$\mathbf{7}$ & 0,578303061 & 4,819192175 & 88,62898851 \\
$\mathbf{8}$ & 0,441636735 & 3,680306126 & 92,30929463 \\
$\mathbf{9}$ & 0,320411387 & 2,670094894 & 94,97938953 \\
$\mathbf{1 0}$ & 0,24803071 & 2,066922585 & 97,04631211 \\
$\mathbf{1 1}$ & 0,220351816 & 1,836265135 & 98,88257725 \\
$\mathbf{1 2}$ & 0,13409073 & 1,11742275 & 100 \\
\hline
\end{tabular}

Nota. Fonte: Dados da pesquisa.

Uma vez definidos os fatores ortogonais, considerou-se significativa a relação dos itens para esses fatores, se as cargas fatoriais ultrapassassem 0.50 (Mingoti, 2005) e se as comunalidades evidenciassem percentuais maiores que 0.50 (Hair et al., 1988). Os resultados mostram o seguinte: (a) Os fatores um e dois contêm quatro itens que atingiram a carga fatorial desejável. Para o primeiro, têm-se os itens benevolência 4 , benevolência 2 , benevolência 3 e integridade 3 , enquanto para o segundo observaram-se os itens integridade 3 , benevolência 1 , integridade 1 e competência 1 . (b) Os fatores três e quatro apresentaram somente dois itens que atenderam a este parâmetro, sendo competência 4 e integridade 4 para o fator três; e competência 3 e competência 2 para o fator quatro. (c) $\mathrm{O}$ item integridade 2 não obteve o valor mínimo de comunalidade. (d) $\mathrm{O}$ item integridade 3 teve carga fatorial superior a $0.50 \mathrm{em}$ dois fatores (fatores $1 \mathrm{e} 2$ ). Em conjunto, tais resultados sinalizam a impossibilidade de desmembrar, exata e distintamente, o construto confiança em integridade, benevolência e competência, o que quer dizer que a característica multidimensional do construto confiança não é claramente reduzida a um número menor de fatores ortogonais; contudo tal resultado não impede a construção de um índice para representar confiança a partir de todos os itens pesquisados. Ressalta-se, outrossim, que não é recomendável elaborar índices sintéticos de segundo nível que venham a representar integridade, benevolência, e competência (ver Tabela 6). 
Tabela 6

Resultados da Análise Fatorial - Cargas fatoriais e Comunalidades

\begin{tabular}{lccccc}
\hline Fatores Varimax & Fator 1 & Fator 2 & Fator 3 & Fator 4 & Comunalidade \\
\hline BENEVOLÊNCIA_4 & $0,811672901^{*}$ & 0,18131087 & 0,053189953 & 0,129843264 & $0,711374974^{* *}$ \\
BENEVOLÊNCIA_2 & $0,764217972^{*}$ & 0,196125917 & 0,075774365 & 0,27541732 & $0,704090929^{* *}$ \\
BENEVOLÊNCIA_3 & $0,689602167 *$ & 0,029347143 & 0,274127025 & $-0,089725252$ & $0,559608649^{* *}$ \\
INTEGRIDADE_3 & $0,608582392^{*}$ & $0,572750417^{*}$ & 0,188224167 & 0,050276745 & $0,736371655^{* *}$ \\
BENEVOLÊNCIA_1 & 0,000178112 & $0,867554036^{*}$ & 0,046642457 & 0,054340634 & $0,757778461^{* *}$ \\
INTEGRIDADE_1 & 0,362871191 & $0,84760821^{*}$ & 0,023940257 & $-0,023072069$ & $0,851220635^{* *}$ \\
COMPETÊNCIA_1 & 0,25583458 & $0,586113104^{*}$ & 0,407836593 & 0,235161073 & $0,63061132^{* *}$ \\
COMPETÊNCIA_4 & 0,087155019 & $-0,078821688$ & $0,788476913 *$ & 0,16360017 & $0,662269715^{* *}$ \\
INTEGRIDADE_4 & 0,202920645 & 0,308441636 & $0,780992205 *$ & 0,131459518 & $0,763543461 * *$ \\
INTEGRIDADE_2 & 0,410207365 & 0,345881279 & 0,426829816 & $-0,075493142$ & 0,475786849 \\
COMPETÊNCIA_3 & 0,042472974 & $-0,020202902$ & 0,142406158 & $0,888703757 *$ & $0,812285994^{* *}$ \\
COMPETÊNCIA_2 & 0,121901525 & 0,120560033 & 0,098396538 & $0,842697965^{*}$ & $0,749216443^{* *}$ \\
\hline Soma Quadrados & 2,519599352 & 2,450787394 & 1,732013969 & 1,711758364 & 8,414159078 \\
\% Variância & 20,99666126 & 20,42322828 & 14,43344975 & 14,26465303 & 70,11799232 \\
\hline
\end{tabular}

Nota. * Cargas fatoriais superiores a $0,50 * *$ Comunalidades superiores a 0,50

Fonte: Dados da pesquisa.

Alternativamente, se em vez de levar em consideração os autovalores superiores a um (o que rendeu um percentual acumulado de variância explicada de 70,11\%), fosse considerado o limite mínimo de $90 \%$ de variância explicada, teríamos um número mínimo de itens necessários à redução da variável confiança igual a oito, no total de 12 itens, o que, na prática, não justificaria a perda de informações dos itens originais (ver anteriormente na Tabela 5). Novamente, conclui-se que não há ganhos estatísticos na redução de variáveis relevantes para confiança.

Dessa forma, decidiu-se prosseguir com a análise de resultados provenientes da estatística inferencial, mantendo-se os 12 itens originalmente testados, e não a sua representação por fatores ortogonais. Para tanto construiu-se um índice sintético para representar o construto confiança, a partir da soma dos 12 itens, com peso igual para todos eles.

Finalmente, o último teste utilizado para validar a amostra e a viabilidade da análise de regressão múltipla buscava verificar a existência de multicolinearidade entre as variáveis, por meio da medida VIF (Hair et al., 1988). Conforme Tabela 7, os quatro modelos de regressão utilizados apresentam resultados bastante baixos dessa medida, todos por volta de um, o que indica que o grau de multicolinearidade entre as variáveis independentes do modelo de regressão é praticamente irrelevante. 
Tabela 7

Resultados do Teste VIF

\begin{tabular}{cc}
\hline Variáveis dependentes da regressão & Valor VIF \\
\hline Custos de informação & 1,0014 \\
Custos de negociação & 1,0115 \\
Custos de implementação & 1,0146 \\
Custos de monitoramento & 1,0460 \\
\hline
\end{tabular}

Nota. Fonte: Dados da pesquisa.

Diante desses parâmetros, que indicam a plausibilidade de se efetuar a análise de regressão múltipla, tem-se, na Tabela 8, o primeiro modelo, que relaciona confiança e custos de informação. Com uma aderência de $24 \%\left(\mathrm{R}^{2}\right.$ ajustado $\left.=0,23995\right)$, os resultados apontam a rejeição da primeira hipótese. Ou seja, ceteris paribus, não existe relação negativa entre confiança e custos de informação. É interessante ressaltar que a variável de controle quantidade de processos de compra apresentou relação significativa e positiva $(\beta=0,23951$; $p$-valor $<0.05)$ com os custos de informação. Isso indica que um número maior de processos de compra está associado a maiores custos de informação.

Tabela 8

Resultados do Modelo de Regressão Linear Múltipla entre as Variáveis Independente e de Controle com os Custos de Informação

\begin{tabular}{lccc}
\hline \multicolumn{4}{c}{ Variáveis com coeficientes significativos (95\%/5\%) } \\
\hline & $\boldsymbol{\beta}$ & $\mathbf{T}$ & P-Valor \\
\hline (Constante) & 6,114694 & 6,282 & 0,000064973 \\
Qtde_Processo_Compra & 0,23951 & 4,019 & 0,000210521 \\
\hline \multicolumn{4}{c}{ Variáveis com coeficientes não significativos (95\%/5\%) } \\
\hline Tempo_Relacionamento & $\boldsymbol{\beta}$ & $\mathbf{T}$ & $\mathbf{P}$-Valor \\
Soma_Confiança & 0,064188178 & 0,501691944 & 0,618277063 \\
Duração_Média_Processo & 0,026940739 & 0,21144814 & 0,833471628 \\
Complex_Produtos & $-0,10953732$ & $-0,827800015$ & 0,412057919 \\
Complex_Negócios & $-0,04215918$ & $-0,326717647$ & 0,745363453 \\
Média_APV & $-0,10399464$ & $-0,814632605$ & 0,419480223 \\
NFuncionários & $-0,14173955$ & $-1,074597577$ & 0,288159453 \\
\hline
\end{tabular}

Nota. $R=0,505751 ; R^{2}=0,255784 ; R^{2}$ Ajustado $=0,23995$

Fonte: Dados da pesquisa.

O segundo modelo da regressão múltipla relaciona confiança e custos de negociação para o teste da hipótese dois. Com nível de aderência de $30 \%\left(\mathrm{R}^{2}\right.$ ajustado $\left.=0,2958\right)$, os resultados não permitem rejeitar tal hipótese, isso porque foi verificada uma associação significativa e negativa entre as duas variáveis testadas $(\beta=-0,3112$; $p$-valor $<0.05)$; portanto os resultados constantes na Tabela 9 sugerem que, ceteris paribus, níveis maiores de confiança estão acompanhados de menores custos de negociação. 
Tabela 9

Resultados do Modelo de Regressão Linear Múltipla entre as Variáveis Independente e de Controle com os Custos de Negociação

\begin{tabular}{|c|c|c|c|}
\hline \multicolumn{4}{|c|}{ Variáveis com coeficientes significativos $(95 \% / 5 \%)$} \\
\hline & $\beta$ & $\mathbf{T}$ & P-Valor \\
\hline (Constante) & 31,1990 & 4,9113 & 0,000022483 \\
\hline Soma_Confiança & -0.3112 & $-2,0763$ & 0,00435 \\
\hline \multicolumn{4}{|c|}{ Variáveis com coeficientes não significativos $(95 \% / 5 \%)$} \\
\hline & $\beta$ & $\mathbf{T}$ & P-Valor \\
\hline Duração_Média_Processo & $-0,029277493$ & $-0,220147527$ & 0,826774519 \\
\hline Tempo_Relacionamento & $-0,018328342$ & $-2,321042444$ & 0,024872992 \\
\hline Qtde_Processo_Compra & $-0,026958961$ & $-0,201763936$ & 0,841031215 \\
\hline NFuncionários & 0,009733156 & 0,072814237 & 0,942283942 \\
\hline Média_APV & $-0,012781593$ & $-0,09321843$ & 0,926153213 \\
\hline Complex_Negócios & 0,046256487 & 0,309932702 & 0,758075344 \\
\hline Complex_Produtos & $-0,067172401$ & $-0,470325186$ & 0,640445771 \\
\hline
\end{tabular}

Nota. $R=0,5826 ; R^{2}=0,3394 ; R^{2}$ Ajustado $=0,2958$

Fonte: Dados da pesquisa.

A hipótese três sugere uma relação negativa entre confiança e custos de implementação. Com $21 \%$ de aderência $\left(\mathrm{R}^{2}\right.$ ajustado $\left.=0,2070\right)$, os resultados do terceiro modelo de regressão múltipla apontam a rejeição dessa hipótese (ver Tabela 10). Isso significa que, ceteris paribus, níveis maiores de confiança não estão associados a menores custos de implementação. Cabe salientar que as variáveis de controle complexidade de negócios e tamanho da firma apresentaram relações significativas e positivas com tais custos (respectivamente $\beta=1,64033$; $p$-valor $<0.05$ e $\beta=0,0015$; $p$-valor $<0.05$ ).

Tabela 10

Resultados do Modelo de Regressão Linear Múltipla entre as Variáveis Independente e de Controle com os Custos de Implementação

\begin{tabular}{lccc}
\hline \multicolumn{4}{c}{ Variáveis com coeficientes significativos (95\%/5\%) } \\
\hline & $\square$ & $\mathbf{T}$ & P-Valor \\
\hline (Constante) & 11,8771 & 4,9289 & 0,0000235 \\
Complex_Negócios & 1,64033 & 2,432623434 & 0,018978987 \\
NFuncionários & 0,0015 & 3,2048 & 0,0025 \\
\hline \multicolumn{4}{c}{ Variáveis com coeficientes não significativos (95\%/5\%) } \\
\hline \multicolumn{4}{c}{$\mathbf{T}$} \\
\hline Soma_Confiança & $\square$ & $-0,782021023$ & 0,438695332 \\
Complex_Produtos & $-0,09816705$ & 0,208581316 & 0,835807888 \\
Média_APV & 0,032849083 & 0,162315514 & 0,871854738 \\
Qtde_Processo_Compra & 0,019563193 & $-1,285415101$ & 0,2058600816 \\
Tempo_Relacionamento & $-0,15582353$ & 2,370301666 & 0,22440772 \\
Duração_Média_Processo & 0,00958063 & 1,986906616 & 0,49481378 \\
\hline
\end{tabular}

Nota. $R=0,4899 ; R^{2}=0,2400 ; R^{2}$ Ajustado $=0,2070$

Fonte: Dados da pesquisa. 
Finalmente, o último modelo de regressão múltipla permitiu testar a hipótese quatro, que sugere uma relação negativa entre confiança e custos de monitoramento. Com uma aderência de $28 \%\left(\mathrm{R}^{2}\right.$ ajustado $=0,279$ ), os resultados não permitem rejeitar tal hipótese, ou seja, em condições ceteris paribus, existe uma relação negativa e significativa entre confiança e custos de monitoramento ( $\beta=$ 0,60824; p-valor <0.05) (ver Tabela 11). Nesse modelo, verificou-se ainda uma relação positiva e significativa entre duração média de processos e custos de monitoramento ( $\beta=1,8631684$; pvalor $<0.05)$.

Tabela 11

Resultados do Modelo de Regressão Linear Múltipla entre as Variáveis Independente e de Controle com os Custos de Monitoramento

\begin{tabular}{lccc}
\hline \multicolumn{4}{c}{ Variáveis com coeficientes significativos (95\%/5\%) } \\
\hline \multirow{4}{*}{ Constante) } & $\boldsymbol{\beta}$ & $\mathbf{T}$ & $\mathbf{P}$-Valor \\
Duração_Média_Processo & 28,4232701 & 4.66600689 & 0,000000553 \\
Soma Confiança & 1,8631684 & 2,85886416 & 0,012994513 \\
\hline \multicolumn{4}{c}{ Variáveis com coeficientes não significativos (95\%/5\%) } \\
\hline Complex_Produtos & $\boldsymbol{\beta}$ & $\mathbf{T}$ & $\mathbf{P}$-Valor \\
Tempo_Relacionamento & 0,143745077 & 0,890439983 & 0,378072831 \\
Complex_Negócios & 0,08695311 & 0,713918093 & 0,479047998 \\
Qtde_Processo_Compra & 2,295221393 & 1,967853143 & 0,055265832 \\
NFuncionários & 0,037436755 & 0,285397869 & 0,776678556 \\
Média_APV & $-0,11457121$ & $-0,928389053$ & 0,358271002 \\
\hline
\end{tabular}

Nota. $R=0,570064 ; R^{2}=0,3249463 ; R^{2}$ Ajustado= 0,2799651

Fonte: Dados da pesquisa.

\section{Conclusão}

O teste empírico das hipóteses de pesquisa sinaliza uma relação negativa e significativa entre confiança e custos de negociação e de monitoramento; no entanto não foram observadas associações significativas entre confiança e custos de informação e de implementação. Considerando que os custos de negociação e de monitoramento são apontados como os principais tipos de custos de transação incorridos pela AUTOMAKER, no relacionamento com os seus principais fornecedores, pode-se dizer que, de maneira geral, esses resultados vão ao encontro de uma literatura crescente, tanto no meio acadêmico internacional (Gambeta, 1998; Kramer, 2006; McEvily et al., 2003), quanto no nacional (Cunha \& Melo, 2006; Lazzarini et al., 2005), para ressaltar a confiança como importante mecanismo de governança em relacionamentos interorganizacionais. Tal como mensurada neste trabalho, a confiança extrapola as fronteiras da confiança calculada (Williamson, 1993), ao incorporar os elementos de honradez, benevolência e integridade (Mayer et al., 1995). Essa confiança, quando presente em relacionamentos interorganizacionais, pode significar benefícios econômicos para os atores (Smith \& Aldrich, 1991), na medida em que está associada negativamente a alguns tipos de custos de transação (Dyer, 1997). 
De maneira específica, apesar de tratar de um estudo de caso, esses resultados podem ser vistos como um refinamento, ainda que modesto, das conclusões alcançadas por Dyer e Chuo (2003), na medida em que esses autores encontraram correlações significativas e negativas somente entre confiança e custos de transação ex post. Como efetuamos, a partir de Williamson (1985), a decomposição dos custos de transação ex ante em custos de informação e de negociação e dos custos de transação ex post em custos de implementação e de monitoramento, nos deparamos com um quadro mais complexo, em termos da associação entre confiança e custos de transação, qual seja: existem correlações significativas e negativas com um tipo de custo ex ante (custos de negociação) e com somente um tipo de custo ex post (custo de monitoramento). Em nível teórico, esses resultados indicam que tanto os dois tipos de custos de transação ex ante quanto os dois tipos de custos de transação ex post não são perfeitamente correlacionados entre si, tal como sugere, mesmo que implicitamente, Dyer e Chuo (2003). Acredita-se, portanto, que estudos futuros que tenham como objetivo analisar a relação entre confiança e custos de transação possam beneficiar-se de uma maior decomposição do construto custos de transação (Williamson, 1985).

Esses resultados suscitam também implicações gerenciais para a indústria automotiva, notadamente para a AUTOMAKER. Considerando que essas firmas, ao longo dos últimos anos, têm investido pesadamente em mecanismos de monitoramento de seus principais fornecedores e elaborado sofisticados aparatos de salvaguardas (Dyer, 1997; Sako \& Helper, 1998), ou seja, em ações que tendem a aumentar o montante de custos de transação, os resultados deste trabalho apontam uma alternativa de relacionamento com esses fornecedores, ao estimular atividades que reforcem a confiança entre montadora e fornecedores. Tal recomendação não é trivial, principalmente se considerarmos que os custos de produção da indústria automotiva se encontram em limiar inferior. Em outras palavras, se as alternativas para redução de custos de produção nessa indústria estão se exaurindo, parece existir espaço, especialmente no caso da AUTOMAKER, para a redução de custos de transação, notadamente os de negociação e os de monitoramento, por meio de mecanismos relacionais de governança, entre os quais se destaca a confiança. Isso não quer dizer substituir os contratos formais entre a montadora e seus principais fornecedores, mas levar adiante a proposta que considera confiança e contratos formais como mecanismos complementares de governança em relacionamentos interorganizacionais (Puranam \& Vanneste, 2009).

A despeito dessas contribuições, o presente trabalho apresenta algumas limitações. A primeira diz respeito à escolha do método, o qual, pela sua própria natureza, inibe qualquer possibilidade de generalização estatística. Cabe ressaltar que esse tipo de generalização deve ser mesmo evitado para a base total de fornecedores da AUTOMAKER. A segunda limitação se refere ao tamanho da amostra que, apesar de ser suficiente para os testes estatísticos utilizados (Hair et al., 1988), pode se considerada pequena. A terceira limitação diz respeito aos resultados da regressão múltipla, os quais foram balizados por níveis de aderência de $\mathrm{R}^{2}$ relativamente baixos: entre $20 \%$ e $30 \%$. Outra limitação é que a associação entre confiança e custos de transação ex ante e ex post, tal como testada neste trabalho, não implica causalidade; portanto, não se sabe se níveis maiores de confiança podem ser vistos como antecedentes de menores custos de transação ou vice-versa. Essa direção de causalidade entre esses dois construtos é, a nosso ver, um dos principais desafios de pesquisas futuras em relacionamentos interorganizacionais, pois requer a coleta de dados quantitativos longitudinais o que, por si só, é tarefa árdua.

Uma limitação que também merece ser comentada diz respeito ao fato de tratarmos a confiança como exógena ao relacionamento interorganizacional. Isso significa que medimos as consequências e não os antecedentes da confiança. Nesse sentido, sugere-se estudos futuros que lancem luzes sobre os fatores que antecedem a formação de confiança em relacionamentos interorganizacionais. Além disso, apesar de tomarmos como referência o trabalho de Dyer e Chuo (2003), assinalamos que a maneira como mensuramos custos de transação implica uma simplificação do conceito.

Pode-se ainda considerar como limitação deste trabalho o fato de não termos realizado entrevistas semiestruturadas com os compradores da AUTOMAKER, com o intuito de aprofundar os resultados aqui apresentados. Ressalta-se também que não incluímos no nosso modelo de pesquisa a variável poder (Faria, 2007). Na indústria automotiva, onde a montadora detém notavelmente maior 
poder sobre os seus principais fornecedores (Wasti \& Wasti, 2008), acredita-se que esta variável possa ter interferência na relação entre confiança e custos de transação. Estudos futuros poderiam contemplar a possibilidade de testar esses três construtos conjuntamente. Finalmente, cabe destacar que, apesar de o presente trabalho chamar atenção para a importância da confiança na redução dos custos de negociação e de monitoramento e, dessa forma, sugerir às firmas que busquem alternativas para zelar e aumentar a confiança com os fornecedores, não temos elementos para avançar em outras implicações gerenciais. Assim, recomendamos que estudos futuros sejam construídos de tal maneira que possibilitem derivar recomendações para executivos envolvidos com a elaboração, implementação e monitoramento de contratos, bem como fornecer heurísticas de como identificar compradores que sejam potencialmente confiáveis.

\section{Artigo recebido em 20.04.2010. Aprovado em 07.01.2011.}

\section{Agradecimentos}

Agradecemos a Alessandro Miake a assessoria estatística e aos dois pareceristas anônimos a possibilidade de corrigirmos e esclarecermos diversos pontos. Erros remanescentes são de nossa inteira responsabilidade.

\section{Referências}

Argyres, N., \& Mayer, K. (2007). Contract design as a firm capability: an integration of learning and transaction cost perspectives. Academy of Management Review, 32(4), 1060-1077.

Babbie, E. (2005). The basics of social research. Belmont, CA: Thomson Wadsworth.

Barber, B. (1983). The logic and limits of trust. New Brunswick, NJ: Rutgers University Press.

Bromiley, P., \& Cummings, L. (1995). Transaction costs in organizations with trust. In R. Bies, B. Sheppard, \& R. Lewicki (Eds.), Research on negotiation in organizations (Vol. 5, pp. 219-247). Greenwich, CT: JAI Press.

Butler, J. (1991). Toward understanding and measuring conditions of trust: evolution of conditions of trust inventory. Journal of Management, 17(3), 643-663. doi: 10.1177/014920639101700307

Butler, J., \& Cantrell, R. (1984). A behavioural decision theory approach to modelling dyadic trust in superiors and subordinates. Psychological Reports, 55(1), 19-28.

Cai, R. (2004). Trust and transaction costs in industrial districts (Dissertação de mestrado). Virginia Polytechnic Institute e State University, Blacksburg, Virginia, USA.

Coase, R. (1937). The nature of the firm. Economica, 4(16), 386-405.

Cunha, C., \& Melo, M. (2006). A confiança nos relacionamentos interorganizacionais: o campo da biotecnologia em análise. RAE Eletrônica, 5(2). doi: 10.1590/S1676-56482006000200009

Demsetz, H. (1991). The theory of the firm revisited. In O. Williamson \& S. Winter (Eds.), The nature of the firm: origin, evolution and development (pp. 159-178). Oxford: Oxford University Press.

Deutsch, M. (1958). Trust and suspicion. Journal of Conflict Resolution, 2(4), 265-279. doi: $10.1177 / 002200275800200401$

Deutsch, M. (1960). The effect of motivational orientation on trust and suspicion. Human Relations, 13(39), 123-139. doi: 10.1177/001872676001300202 
Deutsch, M. (1962). Cooperation and trust: some theoretical notes. In M. Jones (Ed.), Nebraska symposium on motivation (pp. 275-319). Lincoln, NE: University of Nebraska Press.

Doney, P., \& Cannon, J. (1997). An examination of the nature of trust in buyer-seller relationships. Journal of Marketing, 61(2), 35-51. doi: 10.2307/1251829

Dubois, A., \& Gibbert, M. (2010). From complexity to transparency: managing the interplay between theory, method and empirical phenomena in IMM case studies. Industrial Marketing Management, 39(1), 129-136. doi: 10.1016/j.indmarman.2009.08.003

Dyer, J. (1997). Effective interfirm collaboration: how firms minimize transaction costs and maximize transaction value. Strategic Management Journal, 18(7), 535-556. doi: 10.1002/(SICI)10970266(199708)18:7<535::AID-SMJ885>3.0.CO;2-Z

Dyer, J., Cho, D., \& Chu, W. (1998). Strategic supplier segmentation: the next 'best practice' in supply chain management. California Management Review, 40(2), 57-76.

Dyer, J., \& Chu, W. (2000). The determinants of trust in supplier-automaker relationships in the U.S., Japan and Korea. Journal of International Business Studies, 31(2), 259-285. doi: 10.1057/palgrave.jibs.8490905

Dyer, J., \& Chu, W. (2003). The role of trustworthiness in reducing transaction costs and improving performance: empirical evidence from the United States, Japan, and Korea. Organization Science, 14(1), 57-68. doi: 10.1287/orsc.14.1.57.12806

Elian, S. (1998) Análise de regressão. São Paulo: IME/ USP

Faria, A. (2007). Uma proposta de framework em gerência estratégica de redes verticais [1a Edição Especial]. Revista de Administração Contemporânea, 11, 33-54. doi: 10.1590/S141565552007000500003

Foss, K., \& Foss, N. (2005). Resources and transaction costs: how property rights economics furthers the resource-based view. Strategic Management Journal, 26(6), 541-553. doi: 10.1002/smj.465

Gabarro, J. (1978). The development of trust, influence and expectations. In A. Athos \& J. Gabarro (Eds.), Interpersonal behavior (pp. 290-303). Englewood Cliffs, NJ: Prentice Hall.

Gambetta, D. (1988). Can we trust "trust"? In D. Gambetta (Ed.), Trust: making and breaking cooperative relations (pp. 213-237). New York: Basil Blackwell.

Geyskens, I., Steenkamp, J., \& Kumar (2006). Make, buy, or ally: a transaction cost theory metaanalysis. Academy of Management Journal, 49(3), 519-543.

Giffin, K. (1967). The contribution of studies of source credibility to a theory of interpersonal trust in the communication process. Psychological Bulletin, 68(2), 104-107. doi: 10.1037/h0024833

Gulati, R., \& Nickerson, J. (2008). Interorganizational trust, governance choice, and exchange performance. Organization Science, 19(5), 688-708. doi: 10.1287/orsc.1070.0345

Gulati, R., \& Sytch, R. (2008). Does familiarity breed trust? Revisiting the antecedents of trust. Managerial and Decision Economics, 29(2-3), 165-190. doi: 10.1002/mde.1396

Hair, J., Anderson, R., Tatham, R., \& Black, W. (1988). Multivariate data analysis. New Jersey: Prentice Hall.

Jensen, P., \& Webster, E. (2009). Knowledge management: does capture impede creation? Industrial and Corporate Change, 18(4), 701-727. doi: 10.1093/icc/dtp025

Kramer, R. (2006). Organizational trust: a reader. Oxford: Oxford University Press. 
Larzelere, R., \& Huston, T. (1980). The dyadic trust scale: toward understanding interpersonal trust in close relationships. Journal of Marriage and the Family, 42(3), 595-604. doi: 10.2307/351903

Lazzarini, S., Madalozzo, R., Artes, R., \& Siqueira, J. (2005). Measuring trust: an experiment in Brazil. Economia Aplicada, 9(2), 153-169.

Lazzarini, S., Miller, G., \& Zenger, T. (2008). Dealing with the paradox of embeddedness: the role of contracts and trust in facilitating movement out of committed relationships. Organization Science, 19(5), 709-728. doi: 10.1287/orsc.1070.0336

Lewicki, R., McAllister, D., \& Bies, R. (1998). Trust and distrust: new relationships and realities. Academy of Management Review, 23(3), 438-458. doi: 10.2307/259288

Lewis, J., \& Weigert, A. (1985). Trust as a social reality. Social Forces, 63(4), 967-985. doi: $10.2307 / 2578601$

Macaulay S. (1963). Non-contractual relations in business: a preliminary study. American Sociological Review, 28(1), 55-67. doi: 10.2307/2090458

Macneil, I. (1978). Contracts: adjustment of long-term economic relations under classical, neoclassical, and relational contract law. Northwestern University Law Review, 72(6), 854-905.

Madhok, A. (2002). Reassessing the fundamentals and beyond: Ronald Coase, the transaction cost and resource-based theories of the firm and the institutional structure of production. Strategic Management Journal, 23(6), 535-550. doi: 10.1002/smj.247

Malhotra, N. (2001). Pesquisa de marketing: uma orientação aplicada. Porto Alegre: Bookman.

Mayer, R., Davis, J., \& Schoorman, F. (1995). An integrative model of organizational trust. Academy of Management Review, 20(3), 709-734. doi: 10.2307/258792

Mesquita, L., \& Brush, T. (2008). Untangling safeguard and production coordination effects in longterm buyer-supplier relationships. Academy of Management Journal, 51(4), 785-807. doi: $10.2307 / 20159539$

Mesquita, L., \& Lazzarini, S. (2008). Vertical and horizontal relationships in developing economies: implications for SMEs' access to global markets. Academy of Management Journal, 51(2), 359380. doi: $10.2307 / 20159513$

McEvily, B., Perrone, V., \& Zaheer, A. (2003). Trust as an organizing principle. Organization Science, 14(1), 91-103. doi: 10.1287/orsc.14.1.91.12814

Mingoti, S. (2005). Análise de dados através de métodos de estatística multivariada: uma abordagem aplicada. Belo Horizonte: Editora UFMG.

Moorman, C., Deshpande, R., \& Zaltman, G. (1993). Factors affecting trust in market research relationships. Journal of Marketing, 57(1), 81-101. doi: 10.2307/1252059

Nooteboom, B. (1996). Trust, opportunism and governance: a process and control model. Organization Studies, 17(6), 985-1010. doi: 10.1177/017084069601700605

North, D. (1990). Institutions, institutional change and economic performance. New York: Cambridge University Press.

Pereira, R., \& Luce, F. (2009). Até que os custos os separem: por que clientes e fornecedores estabelecem práticas relacionais para o desenvolvimento de projetos de software? Revista de Administração Contemporânea, 13(4), 545-563. doi: 10.1590/S1415-65552009000400003 
Puranam, P., \& Vanneste, B. (2009). Trust and governance: untangling a tangled view. Academy of Management Review, 34(1), 11-31.

Rea, L., \& Parker, R. (2000). Metodologia de pesquisa: do planejamento à execução. São Paulo: Pioneira.

Robinson, S. (1996). Trust and breach of the psychological contract. Administrative Science Quarterly, 41(4), 574-99.

Rotter, J. (1967). A new scale for the measurement of interpersonal trust. Journal of Personality, 35(4), 651-665. doi: 10.1111/j.1467-6494.1967.tb01454.x

Rotter, J. (1971). Generalized expectancies for interpersonal trust. American Psychologist, 26(5), $443-$ 452. doi: $10.1037 / \mathrm{h} 0031464$

Rotter, J. (1980). Interpersonal trust, trustworthiness, and gullibility. American Psychologist, 35(1), 17. doi: 10.1037/0003-066X.35.1.1

Rousseau, D., Sitkin, S., Burt, R., \& Camerer, C. (1998). Not so different after all: a cross-discipline view of trust. Academy of Management Review, 23(3), 393-404. doi: 10.1111/j.1365246X.2007.03696.X

Sako, M., \& Helper, S. (1998). Determinants of trust in supplier relations: evidence from the automotive industry in Japan and the United States. Journal of Economic Behavior \& Organization, 34(3), 387-417. doi: 10.1016/S0167-2681(97)00082-6

Sitkin, S., \& Roth, N. (1993). Explaining the limited effectiveness of legalistic 'remedies' for trust/distrust. Organizational Science, 4(3), 367-392. doi:10.1287/orsc.4.3.367

Smith, A., \& Aldrich, H., (1991, August). The role of trust in the transaction cost economics framework: transaction contexts and governance structures in U.S. manufacturer - supplier relations. Proceedings of the Academy of Management Meeting, Miami, FL, United States.

Sober, E., \& Wilson, D. (1998). Unto others: the evolution and psychology of unselfish behavior. Harvard, Mass.: Harvard University Press.

Uzzi, B. (1997). Social structure and competition in inter-firm networks: the paradox of embeddedness. Administrative Science Quarterly, 42(1), 35-67. doi: 10.2307/2393808

Vanneste, B., \& Puranam, P. (2010). Repeated interactions and contractual detail: identifying the learning effect. Organization Science, 21(1), 186-201. doi: 10.1287/orsc.1080.0402

Wasti, N., \& Wasti, S. (2008). Trust in buyer-supplier relations: the case of the Turkish automotive industry. Journal of International Business Studies, 39(1), 118-131. doi: 10.1057/palgrave.jibs.8400309

Williamson, O. (1985). The economic institutions of capitalism. New York: The Free Press.

Williamson, O. (1991). Comparative economic organization: the analysis of discrete structural alternatives. Administrative Science Quarterly, 36(2), 269-296.

Williamson, O. (1993). The transaction cost economics and organization theory. Journal of Industrial and Corporate Change, 2(1), 107-156. doi: 10.1093/icc/2.1.107

Williamson, O. (1996). The mechanisms of governance. New York: Oxford University Press.

Yin, R. (1994). Case study research: design and methods. Thousands Oaks: Sage Publications. 
Zaheer, A., McEvily, B., \& Perrone, V. (1998). Does trust matter? Exploring the effects of interorganizational and interpersonal trust on performance. Organization Science, 9(2), 141159. doi: 10.1287/orsc.9.2.141

Zanini, M., Lusk, E., \& Woff, B. (2009). Confiança dentro das organizações da nova economia: uma análise empírica sobre as conseqüências da incerteza institucional. Revista de Administração Contemporânea, 13(1), 72-91. doi: 10.1590/S1415-65552009000100006

Zucker, L. (1986). Production of trust: institutional sources of economic structure, 1840-1920. In B. M. Staw \& L. L. Cummings (Eds.), Research in organizational behavior (Vol. 6, pp. 53-111). Greenwich, CT: JAI Press.

Zylbersztajn, D., \& Sztajn, R. (2005). Direito e economia. Rio de Janeiro: Campus. 